\title{
On the Morawetz-Keel-Smith-Sogge Inequality for the Wave Equation on a Curved Background
}

By

Serge AlinhaC*

\section{Introduction}

In their paper [8], Keel, Smith and Sogge establish an improved standard energy inequality for the wave equation, where the energy right-hand side controls the energy and also

$$
[\log (2+t)]^{-1 / 2}\left(\iint_{0}^{t}(1+r)^{-1}|\partial u|^{2} d x d t^{\prime}\right)^{1 / 2}
$$

They obtain this inequality in space dimension $n=3$ using the strong Huygens principle and clever cut-offs. In particular, the inequality shows that, for energy data, the local energy of the solution in, say, $r \leq 1$, is almost integrable in $t$ (and not just bounded as it is close to the light cone). Similar ideas where introduced long ago by Morawetz [15] and have been also developed by Mochizuki and Matsuyama [12], [14]. This improved energy inequality, which we call Morawetz/KSS inequality from the names of the mathematicians who found it, has been used by them to solve semilinear and quasilinear exterior boundary value problems [8], [9]. It has been also extended to higher dimensions by Metcalfe [12] and used in the context of quasilinear wave equations or systems by Metcalfe [12] and Hidano and Yokoyama [6].

In [2] (developing an idea from [1]), we also proved an improved standard energy inequality, in which the "good derivatives" $T_{i}=\partial_{i}+\left(x_{i} / r\right) \partial_{t}$ are shown to behave better close to the light cone. We display this inequality in two slightly different settings : a pure coordinate approach, and a more geometric

Communicated by T. Kawai. Received February 14, 2005. Revised June 3, 2005.

2000 Mathematics Subject Classification(s): 35L40.

*Département de Mathématiques, Université Paris-Sud, 91405 Orsay, France.

e-mail: serge.alinhac@math.u-psud.fr 
approach in the spirit of Christodoulou and Klainerman [5], Klainerman and Nicolò [10]. This inequality plays also a crucial role in the work of Lindblad and Rodniansky [11], and in Alinhac [3], [4].

Thus it seemed interesting to us to try to obtain both inequalities on a curved background; since many bad terms of one inequality have to be controlled by good terms of the other, both inequalities have to be proved together. The remarkable fact here is that the conditions on the metric to obtain one or the other inequality are essentially the same, thus allowing relatively light assumptions. In this paper, using a multiplier technique in the spirit of the original Morawetz paper [13] and of [12], [14], we present two versions (coordinate and geometric) of this inequality. It turns out that very little decay of the perturbed metric is required close to the light cone or to the $t$-axis, while we still have to require rather strong decay for $r>>t$ or inbetween the $t$-axis and the light cone. We hope that this variable coefficients extension will turn out to be useful for nonlinear problems as well.

\section{$\S 1$. Perturbed Wave Equations : the Coordinate Approach}

Let us consider an operator with real coefficients in $\mathbf{R}_{x}^{n} \times \mathbf{R}_{t}(n \geq 3)$

$$
P u \equiv \partial_{t}^{2} u-\Sigma_{1 \leq i, j \leq n} g^{i j} \partial_{i j}^{2} u+\Sigma_{0 \leq \alpha \leq n} a^{\alpha} \partial_{\alpha} u,
$$

where as usual

$$
\begin{aligned}
x_{0}=t, x & =\left(x_{1}, \ldots, x_{n}\right), r=|x|, x=r \omega, \omega_{0}=-1, \\
\sigma & =\left(1+(r-t)^{2}\right)^{1 / 2}=<r-t>.
\end{aligned}
$$

In the following, we will sometimes omit the sum sign, and write for instance $g^{i j} \partial_{i j}^{2} u$ instead of $\Sigma g^{i j} \partial_{i j}^{2} u$, etc. We denote

$$
g^{i j}=\delta^{i j}+\gamma^{i j}, \gamma^{i j}=\gamma^{j i}, c^{2}=\Sigma g^{i j} \omega_{i} \omega_{j}=1+\Sigma \gamma^{i j} \omega_{i} \omega_{j}, c>0,
$$

and assume, for some constant $0 \leq K<1$,

$$
\left|\Sigma \gamma^{i j} \xi_{i} \xi_{j}\right| \leq K|\xi|^{2} .
$$

For a real $u$ we define the energy

$$
E(u)(t)=E(t)=\int\left(\left(\partial_{t} u\right)^{2}+\left|\partial_{x} u\right|^{2}\right) d x .
$$

We will also use the standard Lorentz fields $Z$

$$
\partial_{\alpha}, R=x \wedge \partial_{x}, H_{i}=t \partial_{i}+x_{i} \partial_{t}, S=t \partial_{t}+r \partial_{r}
$$


and the special derivatives

$$
T_{i}=\partial_{i}+\omega_{i} \partial_{t}, T_{0}=0
$$

which span the tangent space to the outgoing cones $r-t=C$. Remark that we have the relations

$$
\begin{aligned}
\partial_{r} & =\Sigma \omega_{i} \partial_{i}, \partial_{i}=\omega_{i} \partial_{r}-(\omega \wedge R / r)_{i} \\
(t+r)\left(\partial_{t}+\partial_{r}\right) & =S+\Sigma \omega_{i} H_{i}, R / r=t^{-1} \omega \wedge H \\
T_{i} & =\left(\partial_{i}-\omega_{i} \partial_{r}\right)+\omega_{i}\left(\partial_{t}+\partial_{r}\right) .
\end{aligned}
$$

These imply the pointwise estimate

$$
\left|T_{i} u\right| \leq C(1+t+r)^{-1} \Sigma|Z u|,
$$

while the following estimate follows from $[6]$ (p. 118)

$$
|\partial u| \leq C \sigma^{-1} \Sigma|Z u|
$$

Theorem 1. Assume that the coefficients of $P$ satisfy, for some $\eta>0$,

$$
\begin{aligned}
|\gamma| \leq C(1+t+r)^{-\eta},|a|+|\partial \gamma| & \leq C(1+t+r)^{\frac{1}{2}-\eta}(1+r)^{-1}<r-t>^{-1 / 2}, \\
\left|\partial^{2} \gamma\right| & \leq C(1+t+r)^{\frac{3}{2}-\eta}(1+r)^{-2}<r-t>^{-3 / 2},
\end{aligned}
$$

and, for $(1+t) / 2 \leq r \leq 2(1+t)$ and all fields $Z$,

$$
|Z \gamma| \leq C(1+t)^{-\eta},|Z \partial \gamma| \leq C(1+t)^{-\frac{1}{2}-\eta}<r-t>^{-1 / 2} .
$$

Then, for any $\epsilon>0$, there exists $C_{\epsilon}$ such that, for all $t \geq 0$ and all $u$ sufficiently vanishing at infinity, we have the inequality

$$
\begin{aligned}
& E(t)+\Sigma \int_{D_{t}} \sigma^{-1}[\log (1+\sigma)]^{-1-\epsilon}\left|T_{i} u\left(x, t^{\prime}\right)\right|^{2} d x d t^{\prime} \\
& \quad+\Sigma \int_{D_{t}}(1+r)^{-1}[\log (2+r)]^{-1-\epsilon}\left|\partial_{\alpha} u\left(x, t^{\prime}\right)\right|^{2} d x d t^{\prime} \\
& \quad+\Sigma \int_{D_{t}} \frac{1}{(1+r)}\left|\partial_{i} u-\omega_{i} \partial_{r} u\right|^{2} d x d t^{\prime}+\int_{D_{t}} \frac{u^{2}}{r(1+r)^{2}[\log (2+r)]^{1+\epsilon}} d x d t^{\prime} \\
& \leq C_{\epsilon} E(0)+C_{\epsilon} \int_{D_{t}}|P u|\left[\left|\partial_{t} u\right|+\left|\partial_{r} u+\frac{n-1}{2} u / r\right|\right] d x d t^{\prime} \\
& \quad+C_{\epsilon} \int_{0}^{t} A\left(t^{\prime}\right) E\left(t^{\prime}\right) d t^{\prime}
\end{aligned}
$$


Here, $D_{t}$ is the strip

$$
D_{t}=\left\{\left(x, t^{\prime}\right), 0 \leq t^{\prime} \leq t\right\}
$$

while the amplification factor $A$ is

$$
A(t)=\left|\sigma^{-1}(c-1)\right|_{L_{x}^{\infty}}+\left|\partial_{t} c\right|_{L_{x}^{\infty}}+\left|\sigma^{1+\epsilon} \partial_{t}^{2} c\right|_{L_{x}^{\infty}}+\left|a^{\alpha} \omega_{\alpha}\right|_{L_{x}^{\infty}}
$$

It is understood here that the $L^{\infty}$ norms in the definition of $A$ are taken only for $(1+t) / 2 \leq r \leq 2(1+t)$.

Proof. In the whole paper, we will distinguish the three regions

$$
I=\{r \leq(1+t) / 2\}, I I=\{(1+t) / 2 \leq r \leq 2(1+t)\}, I I I=\{r \geq 2(1+t)\} .
$$

We use $\epsilon>0$ to denote any strictly positive number, which may vary from one line to another.

1. We have to revisit the proof of Theorem 1 of [2], since the assumptions on $\gamma$ are now weaker. We have, with $p=b(r-t)$,

$$
\begin{aligned}
2 e^{p} P u \partial_{t} u= & \partial_{t}\left[e^{p}\left(\left(\partial_{t} u\right)^{2}+g^{i j}\left(\partial_{i} u\right)\left(\partial_{j} u\right)\right)\right]+\Sigma \partial_{i}(\ldots)+e^{p} Q \\
Q= & b^{\prime} g^{i j} T_{i} u T_{j} u-\partial_{t} \gamma^{i j} T_{i} u T_{j} u+2 T_{i} \gamma^{i j} T_{j} u \partial_{t} u+2 a^{i} T_{i} u \partial_{t} u \\
& +\left(\partial_{t} u\right)^{2}\left[-2 T_{i} \gamma^{i j} \omega_{j}+\partial_{t} \gamma^{i j} \omega_{i} \omega_{j}-2 a^{\alpha} \omega_{\alpha}-b^{\prime} \gamma^{i j} \omega_{i} \omega_{j}\right] .
\end{aligned}
$$

We choose here $b^{\prime}(s)=C_{0}<s>^{-1}[\log (1+<s>)]^{-1-\epsilon}$.

a. In region $\mathrm{I}$,

$$
|a|+|\partial \gamma|+\left|b^{\prime} \gamma\right| \leq C(1+r)^{-1}(1+t)^{-\epsilon},
$$

so that

$$
\int_{I} e^{p} Q d x d t^{\prime} \geq \int_{I} e^{p} b^{\prime} g^{i j} T_{i} u T_{j} u d x d t^{\prime}-C \int_{I} \frac{|\partial u|^{2}}{1+r}\left(1+t^{\prime}\right)^{-\epsilon} d x d t^{\prime} .
$$

b. In region III,

$$
|a|+|\partial \gamma|+\left|b^{\prime} \gamma\right| \leq C(1+r)^{-1-\epsilon}
$$

so that

$$
\int_{I I I} e^{p} Q d x d t^{\prime} \geq \int_{I I I} e^{p} b^{\prime} g^{i j} T_{i} u T_{j} u d x d t^{\prime}-C \int_{I I I}\left(1+t^{\prime}\right)^{-1-\epsilon}|\partial u|^{2} d x d t^{\prime} .
$$

c. In region II, with a small $\epsilon^{\prime}$ to be chosen later,

$$
(|a|+|\partial \gamma|)|T u \partial u| \leq C \epsilon^{\prime} \sigma^{-1-\epsilon}(T u)^{2}+\left(C / \epsilon^{\prime}\right)(1+t)^{-1-\epsilon}(\partial u)^{2} .
$$


On the other hand, $|T \gamma| \leq C(1+t)^{-1-\epsilon}$, hence finally for $\epsilon^{\prime}>0$ small enough

$$
\int_{I I} e^{p} Q \geq(1 / 2) \int_{I I} e^{p} g^{i j} T_{i} u T_{j} u d x d t^{\prime}-C \int_{I I}\left(1+t^{\prime}\right)^{-1-\epsilon}|\partial u|^{2} d x d t^{\prime} .
$$

Putting together the three inequalities, we obtain

$$
\begin{aligned}
E(t) & +\int_{D_{t}} \sigma^{-1}[\log (1+\sigma)]^{-1-\epsilon}|T u|^{2} d x d t^{\prime} \leq C_{\epsilon}\left\{E(0)+\int_{D_{t}}|P u|\left|\partial_{t} u\right| d x d t^{\prime}\right. \\
& \left.+\int_{I} \frac{|\partial u|^{2}}{1+r}\left(1+t^{\prime}\right)^{-\epsilon} d x d t^{\prime}+\int_{0}^{t}\left(1+t^{\prime}\right)^{-1-\epsilon} E\left(t^{\prime}\right) d t^{\prime}+\int_{0}^{t} A_{1}\left(t^{\prime}\right) E\left(t^{\prime}\right) d t^{\prime}\right\},
\end{aligned}
$$

with

$$
A_{1}(t)=\left|\partial_{t} c\right|+\left|a^{\alpha} \omega_{\alpha}\right|+\left|\sigma^{-1}(c-1)\right| .
$$

2. We turn now to the Morawetz type argument yielding the additional inside control. We compute

$$
\int_{D_{t}} P u \zeta\left(r \partial_{r} u+\frac{n-1}{2} u\right) d x d t^{\prime}
$$

for some $\zeta=\zeta(r)$ to be chosen. For simplicity, we give the proof only for $n=3$, the case $n \geq 4$ being exactly similar and yielding slightly better terms. Integrating by parts as usual, we find

$$
\begin{aligned}
& P u \zeta r \partial_{r} u=\partial_{t}\left(r \zeta \partial_{t} u \partial_{r} u\right)+\Sigma \partial_{i}(\ldots) \\
& +(r \zeta)\left[\partial_{j} \gamma^{i j} \partial_{i} u \partial_{r} u-(1 / 2)\left(\partial_{r} \gamma^{i j}\right) \partial_{i} u \partial_{j} u+a^{\alpha} \partial_{\alpha} u \partial_{r} u\right] \\
& +(1 / 2)\left(3 \zeta+r \zeta^{\prime}\right)\left(\partial_{t} u\right)^{2}-(1 / 2)\left(\zeta-r \zeta^{\prime}\right) g^{i j} \partial_{i} u \partial_{j} u-r \zeta^{\prime} g^{i j} \partial_{i} u\left(\partial_{j} u-\omega_{j} \partial_{r} u\right) .
\end{aligned}
$$

Similarly, we find

$$
P u \zeta u=\partial_{t}\left(\zeta u \partial_{t} u\right)+\Sigma \partial_{i}(\ldots)+\zeta\left[-\left(\partial_{t} u\right)^{2}+g^{i j} \partial_{i} u \partial_{j} u+u a^{\alpha} \partial_{\alpha} u\right]+D u^{2},
$$

where

$$
\begin{aligned}
2 D= & -(1 / r)(r \zeta)^{\prime \prime}-\zeta \partial_{i j}^{2} \gamma^{i j}-2 \zeta^{\prime} \omega_{j} \partial_{i} \gamma^{i j} \\
& +\left(\zeta^{\prime} / r\right)\left(\gamma^{i j} \omega_{i} \omega_{j}-\Sigma \gamma^{i i}\right)-\zeta^{\prime \prime} \gamma^{i j} \omega_{i} \omega_{j} .
\end{aligned}
$$

Adding the two expressions, after some rearrangements, we obtain

$$
\begin{aligned}
& P u \zeta\left(r \partial_{r} u+u\right)=\partial_{t}\left[(r \zeta)\left(\partial_{t} u\right)\left(\partial_{r} u+u / r\right)\right]+\Sigma \partial_{i}(\ldots)+D u^{2}+(1 / 2)(r \zeta)^{\prime} \\
& \quad \times\left[\left(\partial_{t} u\right)^{2}+g^{i j} \partial_{i} u \partial_{j} u\right]-r \zeta^{\prime} g^{i j}\left(\partial_{i} u-\omega_{i} \partial_{r} u\right)\left(\partial_{j} u-\omega_{j} \partial_{r} u\right) \\
& \quad-r \zeta^{\prime} \gamma^{i j} \partial_{r} u \omega_{i}\left(\partial_{j} u-\omega_{j} \partial_{r} u\right)+(r \zeta) \\
& \quad \times\left[\partial_{j} \gamma^{i j} \partial_{i} u \partial_{r} u-(1 / 2) \partial_{r} \gamma^{i j} \partial_{i} u \partial_{j} u+a^{\alpha} \partial_{\alpha} u \partial_{r} u\right]+\zeta u a^{\alpha} \partial_{\alpha} u
\end{aligned}
$$


3. We choose now

$$
\zeta(r)=(1 / r) \int_{0}^{r} f(2+s) d s, f(\sigma)=\sigma^{-1}(\log \sigma)^{-1-\epsilon} .
$$

It is easy to check the following properties of $\zeta$ :

i) $\zeta>0, r \zeta \leq C$,

ii) $\zeta^{\prime}<0,(1 / C)(1+r)^{-2} \leq-\zeta^{\prime} \leq C(1+r)^{-2}$,

iii) $\zeta^{\prime \prime}>0,(1 / C)(1+r)^{-3} \leq \zeta^{\prime \prime} \leq C(1+r)^{-3}$,

iv) $(r \zeta)^{\prime \prime}=f^{\prime}(2+r)<0$, and

$$
(1 / C)(1+r)^{-2}[\log (2+r)]^{-1-\epsilon} \leq-(r \zeta)^{\prime \prime} \leq C(1+r)^{-2}[\log (2+r)]^{-1-\epsilon} .
$$

4. To control the $u$ terms by the energy, we will use the two following lemma.

Lemma 1.1. For $n \geq 3$ and $u$ vanishing at infinity, we have

$$
\int|u|^{2} r^{-2} d x \leq C \int\left|\partial_{r} u\right|^{2} d x
$$

We have also the identity

$$
\int\left[\left(\partial_{r} u+u / r\right)^{2}+(n-3) u^{2} / r^{2}\right] d x=\int\left(\partial_{r} u\right)^{2} d x .
$$

Lemma 1.2. For $n \geq 3$, u vanishing at infinity, $\nu>1$, and $\lambda \geq 0$, we have

$$
\int_{r \leq \lambda t}|u(x)|^{2}<r-t>^{-\nu} d x \leq C(\lambda)(1+t) \int\left|\partial_{r} u\right|^{2} d x
$$

Proof of the lemma.

a. The inequality of Lemma 1.1 is well known. To prove the identity, we note that

$$
\Sigma \partial_{i}\left(\omega_{i} u^{2} / r\right)=2 u \partial_{r} u / r+u^{2} \Sigma \partial_{i}\left(x_{i} / r^{2}\right),
$$

and this last term is $(n-2) u^{2} / r^{2}$.

b. If $u$ is supported for $r \leq C(1+t)$, an easy adaptation of Lemma 9.1.3 of [3] gives the result of Lemma 1.2. Finally, let $0 \leq \chi(s) \leq 1$ a smooth cut-off 
supported for $s \leq 2$, and being one for $s \leq 1$. From the previous statement applied to $u_{\lambda}(x)=\chi\left(\frac{r}{\lambda(1+t)}\right) u(x)$, we obtain

$$
\int_{r \leq \lambda t}|u|^{2}<r-t>^{-\eta} d x \leq C(1+t) \int\left|\partial_{r} u\right|^{2} d x+C(1+t)^{-1} \int\left(\chi^{\prime}\right)^{2}|u|^{2} d x
$$

and the last integral is less than

$$
C(1+t)^{2} \int|u|^{2} / r^{2} d x \leq C(1+t)^{2} \int\left|\partial_{r} u\right|^{2} d x
$$

by Lemma 1.1, which finishes the proof.

5. We are now in a position to bound the bad terms in (2.5).

a. (region I). The bad terms in $(\partial u)^{2}$ in $(2.5)$ are bounded by $C(1+$ $r)^{-1}(1+t)^{-\epsilon}|\partial u|^{2}$ as in 1.a. Also

$$
\begin{aligned}
\left|\zeta u a^{\alpha} \partial_{\alpha} u\right| & \leq C(1+r)^{-2}(1+t)^{-\epsilon}|u \partial u| \\
& \leq C \epsilon^{\prime} \frac{u^{2}}{(1+r)^{3+\epsilon}}+\left(C / \epsilon^{\prime}\right) \frac{|\partial u|^{2}}{(1+r)}(1+t)^{-\epsilon} .
\end{aligned}
$$

We consider now $D u^{2}$. We have

$$
\left|\zeta^{\prime \prime} \gamma\right| \leq C\left|\zeta^{\prime} / r \gamma\right| \leq \frac{C}{r(1+r)^{2}(1+t)^{\epsilon}},\left|\zeta^{\prime} \partial \gamma\right|+\left|\zeta \partial^{2} \gamma\right| \leq \frac{C}{(1+r)^{3}(1+t)^{\epsilon}} .
$$

Hence, in region I, all bad terms are controlled by

$$
\begin{aligned}
C \epsilon^{\prime} \int_{I} \frac{u^{2}}{r(1+r)^{2+\epsilon}} d x d t^{\prime} & +\left(C / \epsilon^{\prime}\right) \int_{I} \frac{|\partial u|^{2}}{(1+r)\left(1+t^{\prime}\right)^{\epsilon}} d x d t^{\prime} \\
& +C \int_{I} \frac{u^{2}}{r(1+r)^{2}\left(1+t^{\prime}\right)^{\epsilon}} d x d t^{\prime}
\end{aligned}
$$

b. (region III). There, the coefficients of the bad terms in $(\partial u)^{2}$ are all bounded by $C(1+r)^{-1-\epsilon}$. We also have

$$
\left|\zeta u a^{\alpha} \partial_{\alpha} u\right| \leq C(1+r)^{-2-\epsilon}|u \partial u| \leq C(1+t)^{-1-\epsilon}|\partial u|^{2}+C(1+t)^{-1-\epsilon} u^{2} / r^{2} .
$$

All the bad terms in $D$ are bounded by $C(1+r)^{-3-\epsilon}$. Hence, in region III, all bad terms are controlled by

$$
C \int_{I I I}\left(1+t^{\prime}\right)^{-1-\epsilon}|\partial u|^{2} d x d t^{\prime}+C \int_{I I I}\left(1+t^{\prime}\right)^{-1-\epsilon} u^{2} / r^{2} d x d t^{\prime}
$$


c. (region II). First, we consider the quadratic terms in $\partial u$ in $(r \zeta)[\ldots]$.

We write

$$
\begin{aligned}
\partial_{j} \gamma^{i j} \partial_{i} u= & T_{j} \gamma^{i j} \partial_{i} u-\omega_{j}\left(\partial_{t} \gamma^{i j}\right)\left(T_{i} u-\omega_{i} \partial_{t} u\right) \\
= & \left(\partial_{t} c^{2}\right) \partial_{t} u+T_{j} \gamma^{i j} \partial_{i} u-\omega_{j}\left(\partial_{t} \gamma^{i j}\right) T_{i} u, \\
\partial_{r} \gamma^{i j} \partial_{i} u \partial_{j} u= & -\omega_{i} \partial_{t} u \partial_{r} \gamma^{i j}\left(T_{j} u-\omega_{j} \partial_{t} u\right)+\partial_{r} \gamma^{i j} T_{i} u \partial_{j} u \\
= & {\left[-\partial_{t} c^{2}+\left(\left(\partial_{t}+\partial_{r}\right) \gamma^{i j}\right) \omega_{i} \omega_{j}\right]\left(\partial_{t} u\right)^{2}+\left(\partial_{r} \gamma^{i j}\right) } \\
& \times\left(T_{i} u \partial_{j} u-\omega_{i} \partial_{t} u T_{j} u\right), \\
a^{\alpha} \partial_{\alpha} u \partial_{r} u= & a^{\alpha} T_{\alpha} u \partial_{r} u-a^{\alpha} \omega_{\alpha} \partial_{t} u \partial_{r} u .
\end{aligned}
$$

Thus

$$
\begin{aligned}
& \left|\int_{D_{t}}(r \zeta)\left[\left(\partial_{i} u\right)\left(\partial_{j} \gamma^{i j} \partial_{r} u-(1 / 2) \partial_{r} \gamma^{i j} \partial_{j} u\right)+a^{\alpha} \partial_{\alpha} u \partial_{r} u\right]\right| \\
& \leq\left(C / \epsilon^{\prime}\right) \int_{0}^{t}\left(1+t^{\prime}\right)^{-1-\epsilon} E\left(t^{\prime}\right) d t^{\prime}+C \int_{D_{t}}\left[\left|\partial_{t} c\right|+\left|a^{\alpha} \omega_{\alpha}\right|\right]|\partial u|^{2} d x d t^{\prime} \\
& \quad+C \epsilon^{\prime} \int_{D_{t}} \sigma^{-1-\epsilon}|T u|^{2} d x d t^{\prime},
\end{aligned}
$$

since, in this region,

$$
|a|+|\partial \gamma| \leq C \sigma^{-1 / 2}(1+t)^{-1 / 2-\epsilon},|T \gamma| \leq C(1+t)^{-1-\epsilon} .
$$

The other quadratic terms in $\partial u$ have the good sign, except

$$
F=\left(r \zeta^{\prime}\right) \gamma^{i j} \omega_{i} \partial_{r} u\left(\partial_{j} u-\omega_{j} \partial_{r} u\right),
$$

which is bounded by $C(1+t)^{-1-\epsilon}|\partial u|^{2}$.

We have finally

$$
\left|\zeta u a^{\alpha} \partial_{\alpha} u\right| \leq C(1+t)^{-1-\epsilon} \frac{\sigma^{-1-\epsilon} u^{2}}{(1+t)}+C(1+t)^{-1-\epsilon}|\partial u|^{2} .
$$

We turn now to $D$. We have first

$$
\left|\zeta^{\prime \prime} \gamma\right|+\left|\zeta^{\prime} \gamma / r\right| \leq C(1+t)^{-3-\epsilon} .
$$

Also

$$
\left|\zeta^{\prime} \partial \gamma\right| \leq C \sigma^{-1 / 2}(1+t)^{-5 / 2-\epsilon} \leq C(1+t)^{-1-\epsilon} \frac{\sigma^{-1-\epsilon}}{(1+t)} .
$$

To handle the term $\zeta \partial_{i j}^{2} \gamma^{i j}$, we write

$$
\zeta \partial_{i j}^{2} \gamma^{i j}=\zeta\left[\partial_{t}^{2} c^{2}+T_{i} \partial_{j} \gamma^{i j}-\omega_{i} T_{j} \partial_{t} \gamma^{i j}\right],
$$


and the last two terms can be handled as before since they are bounded by

$$
C \sigma^{-1 / 2}(1+t)^{-5 / 2-\epsilon} \text {. }
$$

Now

$$
\partial_{t}^{2} c^{2}=2\left(\partial_{t} c\right)^{2}+2 c \partial_{t}^{2} c
$$

and $\zeta\left(\partial_{t} c\right)^{2} \leq C \sigma^{-1}(1+t)^{-2-\epsilon}$. We write finally

$$
\begin{aligned}
\int\left|u^{2} \zeta \partial_{t}^{2} c\right| d x & \leq C \int \sigma^{-1-\epsilon}(1+t)^{-1} u^{2}\left|\sigma^{1+\epsilon} \partial_{t}^{2} c\right| d x \\
& \leq C\left|\sigma^{1+\epsilon} \partial_{t}^{2} c\right|_{L^{\infty}} \int|\partial u|^{2} d x
\end{aligned}
$$

by Lemma 1.2 .

d. When integrating over $D_{t}$, we also get boundary terms on $t^{\prime}=0$ and $t^{\prime}=t$

$$
\int(r \zeta)\left(\partial_{t} u\right)\left(\partial_{r} u+u / r\right) d x
$$

By Lemma 1.1, these integrals are control by energy integrals. Putting together all inequalities, and using Lemma 1.1 and Lemma 1.2, we finally get

$$
\begin{aligned}
& \int_{D_{t}} \frac{|\partial u|^{2}}{(1+r)[\log (2+r)]^{1+\epsilon}} d x d t^{\prime}+\Sigma \int_{D_{t}} \frac{1}{1+r}\left|\partial_{i} u-\omega_{i} \partial_{r} u\right|^{2} d x d t^{\prime} \\
& +\int_{D_{t}} \frac{u^{2}}{r(1+r)^{2}[\log (2+r)]^{1+\epsilon}} x d t^{\prime} \leq C_{\epsilon}\left\{\int_{D_{t}}\left|P u \| \partial_{r} u+\frac{n-1}{2} u / r\right| d x d t^{\prime}\right. \\
& +E(t)+E(0)+\int_{I} \frac{u^{2}}{r(1+r)^{2}\left(1+t^{\prime}\right)^{\epsilon}} d x d t^{\prime}+\epsilon^{\prime} \int_{D_{t}} \frac{u^{2}}{(1+r)^{3+\epsilon}} d x d t^{\prime} \\
& +\epsilon^{\prime} \int_{D_{t}} \sigma^{-1-\epsilon}|T u|^{2} d x d t^{\prime}+\left(1 / \epsilon^{\prime}\right) \int_{I} \frac{|\partial u|^{2}}{(1+r)\left(1+t^{\prime}\right) \epsilon} d x d t^{\prime} \\
& \left.+\left(1 / \epsilon^{\prime}\right) \int_{0}^{t}\left(1+t^{\prime}\right)^{-1-\epsilon} E\left(t^{\prime}\right) d t^{\prime}+\int_{0}^{t} A_{2}\left(t^{\prime}\right) E\left(t^{\prime}\right) d t^{\prime}\right\}
\end{aligned}
$$

where

$$
A_{2}(t)=\left|\partial_{t} c\right|+\left|\sigma^{1+\epsilon} \partial_{t}^{2} c\right|+\left|a^{\alpha} \omega_{\alpha}\right|
$$

To finish the argument, we add the above inequality to a large amount of the energy inequality, in order to regain control of $E(t)$. Then we fix $\epsilon^{\prime}>0$ small 
enough to absorb in the left-hand side the terms which are multiplied by $\epsilon^{\prime}$. The terms

$$
\int_{I} \frac{|\partial u|^{2}}{(1+r)\left(1+t^{\prime}\right)^{\epsilon}} d x d t^{\prime}+\int_{I} \frac{u^{2}}{r(1+r)^{2}\left(1+t^{\prime}\right)^{\epsilon}} d x d t^{\prime}
$$

will be absorbed by the left-hand side for $t^{\prime} \geq T_{0}$ big enough. For $t \leq T_{0}$, the corresponding integrals over $D_{t}$ are, by Lemma 1.1, bounded by

$$
C \int_{0}^{t} E\left(t^{\prime}\right) d t^{\prime} .
$$

Using finally Gronwall's Lemma, we obtain the theorem, with $A=A_{1}+$ $A_{2}$.

\section{§2. Wave Equation on a Curved Background : Geometric Approach}

We consider now a split metric on $\mathbf{R}_{x}^{n} \times \mathbf{R}_{t}(n \geq 3)$

$$
g=-d t^{2}+g_{i j} d x^{i} d x^{j}
$$

where as usual $x_{0}=t$, greek indices run from zero to $\mathrm{n}$ and latin indices run from 1 to $n$. We use the standard notation of geometry and assume as before

$$
g^{i j}=\delta^{i j}+\gamma^{i j},\|\gamma\| \leq K, K<1,
$$

where ||.|| denotes the operator norm associated to the standard euclidean norm on $\mathbf{R}^{n}$. We assume, as in part III of [3], that the standard spheres, defined by $t=t_{0}, r=|x|=r_{0}$, play an essential role in our problem (this is what we call a quasiradial situation ). Defining

$$
T=-\nabla t=\partial_{t}, c^{2}=<\nabla r, \nabla r>=g^{i j} \omega_{i} \omega_{j}, N=(1 / c) \nabla r,
$$

we will use the null frame

$$
e_{1}, \ldots, e_{n}, L_{1}=T-N, L=T+N,
$$

where the $e_{j}$ 's are an orthonormal basis on the spheres. We will use the notation $\not \nabla$ for the rotation part of $\nabla u$, and write

$$
|\not \nabla|^{2}=\Sigma e_{a}(u)^{2}
$$

Recall also that the second fundamental form $k(X, Y)=-\left\langle D_{X} T, Y\right\rangle$ of the hypersurfaces

$$
\Sigma_{t}=\{(x, t)\}
$$


satisfies

$$
k_{i j}=-1 / 2 \partial_{t} g_{i j}, k(N, N)=\partial_{t} c / c .
$$

We also use the second fundamental form $\theta(X, Y)=<D_{X} N, Y>$ of the standard spheres in $\Sigma_{t}$. The traces of $k$ and $\theta$ will be denoted by

$$
\bar{k}=\Sigma k_{a a}+k_{N N}, \bar{\theta}=\Sigma \theta_{a a} .
$$

We define the energy at time $t$ to be

$$
E(t)=1 / 2 \int_{\Sigma_{t}}\left[(T u)^{2}+(N u)^{2}+\Sigma\left(e_{a} u\right)^{2}\right] d v .
$$

We state the analogue of Theorem 1 for the d'Alembertian $\square$ associated to $g$. Again, as in 1., we distinguish three zones

$$
I=\{r \leq(1+t) / 2\}, I I=\{(1+t) / 2 \leq r \leq 2(1+t)\}, I I I=\{r \geq 2(1+t)\} .
$$

Theorem 2. Assume the following for the metric $g$ : for some $\eta>0$,

$$
\begin{aligned}
& \text { (Region I) } \quad|\gamma| \leq C(1+t)^{-\eta},|\partial \gamma| \leq C(1+r)^{-1}(1+t)^{-\eta} \text {, } \\
& \text { (Region III) } \quad|\gamma| \leq C(1+r)^{-\eta},|\partial \gamma| \leq C(1+r)^{-1-\eta} \text {, } \\
& (\text { Region II })(\mathbf{1}) \quad|\bar{k}|+|\bar{\theta}-(n-1) /(r c)|+\Sigma\left|k_{a N}\right|+\left|e_{a}(c)\right| \\
& \leq C \sigma^{-1 / 2}(1+t)^{-\frac{1}{2}-\eta} \\
& (\text { Region II })(2) \quad \Sigma_{a \neq b}\left|\theta_{a b}\right|+\Sigma\left|k_{a b}\right|+\left|\theta_{a a}-1 /(r c)\right| \leq C \sigma^{-1-\eta} \text {. }
\end{aligned}
$$

Then, for any $\epsilon>0$, there exists $C_{\epsilon}$ such that, for all $t \geq 0$ and all $u$ sufficiently vanishing at infinity, we have the inequality

$$
\begin{aligned}
E(t) & +\int_{D_{t}} \sigma^{-1}[\log (1+\sigma)]^{-1-\epsilon}\left((L u)^{2}+|\not \nabla u|^{2}\right) d v d t^{\prime} \\
& +\int_{D_{t}}(1+r)^{-1}[\log (2+r)]^{-1-\epsilon}\left((T u)^{2}+(N u)^{2}\right) d v d t^{\prime} \\
& +\int_{D_{t}} \frac{|\not \nabla u|^{2}}{1+r} d v d t^{\prime}+\int_{D_{t}} \frac{u^{2}}{r(1+r)^{2}[\log (2+r)]^{1+\epsilon}} d v d t^{\prime} \\
& \leq C_{\epsilon} E(0)+C_{\epsilon} \int_{D_{t}}|\square u|\left[|T u|+\left|N u+\frac{n-1}{2} u / r\right|\right] d v d t^{\prime} \\
& +C_{\epsilon} \int_{0}^{t} A\left(t^{\prime}\right) E\left(t^{\prime}\right) d t^{\prime} .
\end{aligned}
$$

The amplification factor is here

$$
A(t)=\left|\sigma^{-1}(c-1)\right|_{L_{x}^{\infty}}+\left|\partial_{t} c\right|_{L_{x}^{\infty}}+|N c|_{L_{x}^{\infty}} .
$$


Remark. We note the absence of second order derivatives in $A$. On the other hand, since we did not make an assumption about $Z \gamma$, we have to include the term $N c$ in $A$.

Proof. In regions I and III (the less sensitive regions), we made the same non-geometric assumptions as in Theorem 1, and the terms will be treated in a very similar way. In the following, we will concentrate therefore mostly on region II.

1. We quickly revisit the proof of Theorem 4 of [2]. The energy terms in regions I and III cause no problems and are handled exactly as in the proof of Theorem 1. In region II, the term $k_{N N}|\partial u|^{2}$ is taken care of by the definition of $A$. Since

$$
\Sigma k_{a a}=\bar{k}-k_{N N}
$$

we can replace assumption (1.1) $)_{a}$ of Theorem 4 of [2] by our stronger assumption (1), and the energy inequality holds, with the same error terms as in the proof of Theorem 1.

2. a. As in part one, we will compute $\int_{D_{t}} \square u \zeta\left(r N u+\frac{n-1}{2} u\right) d v d t^{\prime}$ for the same $\zeta$, and take $n=3$ to simplify. We recall first the general formula

$$
\square u X u=-(1 / 2) Q^{\alpha \beta} \pi_{\alpha \beta}+D^{\alpha} K_{\alpha},
$$

where $X=X^{\alpha} \partial_{\alpha}$ is any field,

$$
Q_{\alpha \beta}=\partial_{\alpha} u \partial_{\beta} u-\frac{1}{2} g_{\alpha \beta}<\nabla u, \nabla u>
$$

$K_{\alpha}=Q_{\alpha \beta} X^{\beta}$ and $\pi$ stands for the deformation tensor of $X$ defined by

$$
{ }^{(X)} \pi_{\alpha \beta}=D_{\alpha} X_{\beta}+D_{\beta} X_{\alpha}
$$

Writing $\tilde{\zeta}=r \zeta$, we also have the formula

$$
{ }^{\left(\tilde{\zeta}^{N)}\right.} \pi_{X Y} \equiv \tilde{\pi}_{X Y}=\tilde{\zeta}^{(N)} \pi_{X Y}+X \tilde{\zeta}<N, Y>+Y \tilde{\zeta}<N, X>.
$$

We use now the above formula and express the double trace with the help of our frame and its dual frame $e_{a},-1 / 2 L,-1 / 2 L_{1}$; we find, with $\pi={ }^{(N)} \pi$,

$$
\begin{aligned}
-2\left[\square u \tilde{\zeta} N u-D^{\alpha} K_{\alpha}\right]= & 1 / 4 Q_{L_{1} L_{1}} \tilde{\pi}_{L L}+1 / 4 Q_{L L} \tilde{\pi}_{L_{1} L_{1}} \\
& +1 / 2 Q_{L L_{1}} \tilde{\pi}_{L L_{1}}-Q_{L_{1} a} \tilde{\pi}_{L a}-Q_{L a} \tilde{\pi}_{L_{1} a}+Q_{a b} \tilde{\pi}_{a b}
\end{aligned}
$$


Now

$$
\begin{aligned}
\tilde{\pi}_{L L} & =\tilde{\zeta} \pi_{L L}+2 L \tilde{\zeta}, \tilde{\pi}_{L_{1} L_{1}}=\tilde{\zeta} \pi_{L_{1} L_{1}}-2 L_{1} \tilde{\zeta} \\
\tilde{\pi}_{L L_{1}} & =\tilde{\zeta} \pi_{L L_{1}}-2 N \tilde{\zeta}
\end{aligned}
$$

the other components of $\tilde{\pi}$ being simply those of $\pi$ multiplied by $\tilde{\zeta}$. Thus

$$
-2[\ldots]=1 / 2 L \tilde{\zeta} Q_{L_{1} L_{1}}-1 / 2 L_{1} \tilde{\zeta} Q_{L L}-N \tilde{\zeta} Q_{L L_{1}}+\tilde{\zeta}\left(Q^{\alpha \beta} \pi_{\alpha \beta}\right) .
$$

b. We have as usual from the definition of $Q$

$$
\begin{aligned}
\nabla u & =(-1 / 2) L_{1} u L-(1 / 2) L u L_{1}+\Sigma e_{a}(u) e_{a}, \\
<\nabla u, \nabla u> & =-L u L_{1} u+|\not \nabla u|^{2}, \\
Q_{L L} & =(L u)^{2}, Q_{L_{1} L_{1}}=\left(L_{1} u\right)^{2}, Q_{L L_{1}}=|\not \nabla u|^{2}, Q_{L a}=L u e_{a}(u), \\
Q_{L_{1} a} & =L_{1} u e_{a}(u), Q_{a b}=e_{a}(u) e_{b}(u)-1 / 2 \delta_{a b}<\nabla u, \nabla u>.
\end{aligned}
$$

Hence

$$
-2[\ldots]=(1 / 2) N \tilde{\zeta}\left(L_{1} u\right)^{2}+(1 / 2) N \tilde{\zeta}(L u)^{2}-N \tilde{\zeta}|\not \nabla u|^{2}+\tilde{\zeta}\left(Q^{\alpha \beta} \pi_{\alpha \beta}\right)
$$

c. We can compute explicitly the components of $\pi$ : we have first

$$
\begin{aligned}
<D_{N} N, X> & =-N c / c^{2} X(r)+1 / c<D_{N} \nabla r, X> \\
& =-N c / c^{2} X(r)+1 / c<D_{X}(c N), N> \\
& =-N c / c^{2} X(r)+X(c) / c, \\
<D_{T} N, T> & =0,<D_{T} N, e_{a}>=1 / c<D_{a}(c N), T> \\
& =-<D_{a} T, N>=k_{a N}, \\
<D_{a} N, T> & =-<D_{a} T, N>=k_{a N},<D_{a} N, e_{b}>=\theta_{a b} .
\end{aligned}
$$

Thus

$$
\begin{aligned}
1 / 2 \pi_{L L} & =<D_{N} N, T>=T c / c, 1 / 2 \pi_{L_{1} L_{1}}=-T c / c, \pi_{L L_{1}}=<D_{T} N, T>=0, \\
\pi_{L a} & =<D_{T} N, e_{a}>+<D_{N} N, e_{a}>+<D_{a} N, T>=2 k_{a N}+e_{a}(c) / c, \\
\pi_{L_{1} a} & =2 k_{a N}-e_{a}(c) / c, \pi_{a b}=2 \theta_{a b} .
\end{aligned}
$$

d. Replacing these values into the above expression of the trace, we find

$$
\begin{aligned}
-2[\ldots]= & N \tilde{\zeta}\left((T u)^{2}+(N u)^{2}\right)-N \tilde{\zeta}|\not \nabla u|^{2}-2 \tilde{\zeta}(T c / c) T u N u-\tilde{\zeta} \bar{\theta}<\nabla u, \nabla u> \\
& +\tilde{\zeta}\left(-\pi_{L a} L_{1} u e_{a}(u)-\pi_{L_{1} a} L u e_{a}(u)+2 \theta_{a b} e_{a}(u) e_{b}(u)\right) .
\end{aligned}
$$


3. We have

$$
\begin{aligned}
& \operatorname{div}(\zeta u \nabla u)=\zeta u \square u+<\nabla u, \nabla(\zeta u)>=\zeta u \square u+\zeta<\nabla u, \nabla u>+u<\nabla u, \nabla \zeta>, \\
& \operatorname{div}\left(u^{2} \nabla \zeta\right)=u^{2} \square \zeta+2 u<\nabla u, \nabla \zeta>,
\end{aligned}
$$

hence finally

$$
\square u \zeta u=\operatorname{div}\left(\zeta u \nabla u-1 / 2 u^{2} \nabla \zeta\right)-\zeta<\nabla u, \nabla u>+(1 / 2) u^{2} \square \zeta .
$$

4. Putting together both formula, we obtain (still with $\pi={ }^{(N)} \pi$ )

$$
\begin{aligned}
& -\square u \tilde{\zeta}(N u+u / r)=-\operatorname{div}\left(K+\zeta u \nabla u-1 / 2 u^{2} \nabla \zeta\right)+1 / 2 N \tilde{\zeta}\left((T u)^{2}+(N u)^{2}\right) \\
& -1 / 2 N \tilde{\zeta}|\not \nabla u|^{2}+<\nabla u, \nabla u>(\zeta-1 / 2 \tilde{\zeta} \bar{\theta})-\tilde{\zeta}(T c / c) T u N u+(1 / 2) u^{2} \square \zeta \\
& -(1 / 2) \tilde{\zeta}\left(\pi_{L a} L_{1} u e_{a}(u)+\pi_{L_{1} a} L u e_{a} u-2 \Sigma_{a \neq b} \theta_{a b} e_{a}(u) e_{b}(u)\right)+\tilde{\zeta} \Sigma \theta_{a a} e_{a}(u)^{2}
\end{aligned}
$$

5. a. We study now the components of $\theta$. We have

$$
\begin{aligned}
N & =(1 / c) g^{i j} \omega_{i} \partial_{j}, \\
D_{a} N & =e_{a}\left((1 / c) g^{i j} \omega_{i}\right) \partial_{j}+(1 / c) g^{i j} \omega_{i} e_{a}^{k} \Gamma_{k j}^{\alpha} \partial_{\alpha} .
\end{aligned}
$$

Now

$$
e_{a}\left(\omega_{i}\right)=e_{a}\left(x^{i} / r\right)=1 / r e_{a}^{i}, e_{a}(c)=(1 / 2 c) e_{a}\left(g^{i j}\right) \omega_{i} \omega_{j}+(1 / r c) g^{i j} e_{a}^{i} \omega_{j},
$$

and, since the standard scalar product of $e_{a}$ and $\omega$ is zero,

$$
\begin{aligned}
g^{i j} e_{a}^{i} \omega_{j} & =\gamma^{i j} e_{a}^{i} \omega_{j}, \\
D_{a} N & =O(\partial \gamma)+O(\gamma / r)+(1 / r c) \Sigma g^{i j} e_{a}^{i} \partial_{j} .
\end{aligned}
$$

Here and later, the notation $f=O(g)$ means a pointwise bound

$$
|f(x, t)| \leq C|g(x, t)|
$$

with a constant $C$ fixed in the region under consideration.

Thus

$$
\theta_{a b}=O(\partial \gamma)+O(\gamma / r)+(1 / r c) \Sigma g^{i j} e_{a}^{i} g_{j k} e_{b}^{k}
$$

and the last sum is just the standard scalar product of $e_{a}$ and $e_{b}$, which is $\delta_{a b}+O(\gamma)$. Finally

$$
\theta_{a a}=(1 / r c)+O(\partial \gamma)+O(\gamma / r), \bar{\theta}=(2 / r c)+O(\partial \gamma)+O(\gamma / r)
$$


and, if $a \neq b, \theta_{a b}=O(\partial \gamma)+O(\gamma / r)$. From the expressions of $\pi_{L a}$ and $\pi_{L_{1} a}$ we also have

$$
\left|e_{a}(c)\right|+\left|\pi_{L a}\right|+\left|\pi_{L_{1} a}\right|=O(\partial \gamma)+O(\gamma / r)
$$

b. We compute $\square \zeta$ :

$$
\begin{aligned}
\operatorname{div} N & =\bar{\theta}, \operatorname{div} \nabla r=\operatorname{div} c N=c \bar{\theta}+N c, \\
\square \zeta & =\operatorname{div} \zeta^{\prime} \nabla r=\zeta^{\prime}(c \bar{\theta}+N c)+c^{2} \zeta^{\prime \prime} \\
& =\left(c^{2} / r\right)(r \zeta)^{\prime \prime}+c \zeta^{\prime}(N c / c+\bar{\theta}-(2 c / r)) .
\end{aligned}
$$

We have

$$
|(N c / c+\bar{\theta}-2 c / r)|=O(\partial \gamma)+O(\gamma / r) .
$$

6. We now bound the bad terms in (2.2). In regions I and III, thanks to the above estimates, the analysis is exactly the same as in the proof of Theorem 1 , and we omit it. In region II, consider first the gradient terms. We have

$$
2 \zeta-\tilde{\zeta} \bar{\theta}=2 \zeta \frac{c-1}{c}-\tilde{\zeta}(\bar{\theta}-2 /(r c))=O\left(\sigma^{-1}(c-1)\right)+O(|\bar{\theta}-2 /(r c)|) .
$$

The first term is already taken care of by $A$; since $\langle\nabla u, \nabla u\rangle$ contains at least one good derivative $L u$ or $e_{a}(u)$, our assumption (1) gives as usual

$$
|<\nabla u, \nabla u>(\bar{\theta}-2 /(r c))| \leq C \epsilon^{\prime} \sigma^{-1-\epsilon}\left((L u)^{2}+|\not \supset u|^{2}\right)+\left(C / \epsilon^{\prime}\right)(1+t)^{-1-\epsilon}|\partial u|^{2} .
$$

The same discussion applies to the terms containing one good derivative

$$
\pi_{L a} L_{1} u e_{a}(u), \pi_{L_{1} a} L u e_{a}(u) .
$$

For the pure rotation terms, we write

$$
\Sigma \theta_{a a} e_{a}(u)^{2}-1 /(r c)|\not \nabla u|^{2}=\Sigma\left(\theta_{a a}-1 /(r c)\right) e_{a}(u)^{2} .
$$

Thanks to (2), this last sum is bounded by $C \sigma^{-1-\epsilon}|\not \supset u|^{2}$.

Consider now the bad $u^{2}$ terms. They are bounded by

$$
C(1+t)^{-2}|N c|+\sigma(1+t)^{-3}\left|\sigma^{-1}(c-1)\right|+C(1+t)^{-2}|\bar{\theta}-2 /(r c)| .
$$

Using Lemma 1.1, we can control the integral of the first two terms by $\int_{0}^{t} A\left(t^{\prime}\right)$ $E\left(t^{\prime}\right) d t^{\prime}$. The last term is less than

$$
C(1+t)^{-2} \sigma^{-1 / 2}(1+t)^{-1 / 2-\epsilon} \leq C\left(\sigma^{-1-\epsilon}(1+t)^{-1}\right)(1+t)^{-1-\epsilon}
$$


and, using Lemma 1.2, its integral can be bounded by $\int_{0}^{t}\left(1+t^{\prime}\right)^{-1-\epsilon} E\left(t^{\prime}\right) d t^{\prime}$.

7. It remains to consider the boundary integrals which are less than

$$
\int\left|\partial_{t} u \| N u+u / r\right| d v \leq \int\left|\partial_{t} u\right|^{2} d v+\int|N u|^{2} d v+\int u^{2} / r^{2} d v+2 \int u N u / r d v \text {. }
$$

We remark that

$$
\begin{aligned}
\operatorname{div}\left(\left(u^{2} / r\right) N\right) & =\left(u^{2} / r\right) \bar{\theta}+2 u N u / r \\
& =(2 / c)\left(u^{2} / r^{2}\right)+\left(u^{2} / r\right)(\bar{\theta}-2 /(r c))+2 u N u / r .
\end{aligned}
$$

In regions I or III, the integral of $\left(u^{2} / r\right) \bar{\theta}$ is less than that of $u^{2} / r^{2}$, and we can use Lemma 1.1. In region II,

$$
\left(u^{2} / r^{2}\right)|\bar{\theta}-2 /(r c)| \leq u^{2} \sigma^{-1-\epsilon}(1+t)^{-1}
$$

and we use Lemma 1.2 to conclude.

The rest of the proof is exactly the same as in the proof of Theorem 1.

\section{References}

[1] Alinhac, S., The Null condition for quasilinear wave equations in two space dimensions I, Invent. Math., 145 (2001), 597-618.

[2] $\_$, Remarks on Energy Inequalities for Wave and Maxwell Equations on a Curved Background, Math. Ann., 329 (2004), 707-722.

[3] Soc. Math. France, 133 (2005), 419-458.

[4] , An Example of Blowup at Infinity for a Quasilinear Wave Equation, Astérisque, No. 284 (2003), 1-91.

[5] Christodoulou, D. and Klainerman, S., The global nonlinear stability of the Minkowski space, Princeton Math. Series, 41, Princeton Univ. Press, Princeton, NJ, 1993.

[6] Hidano, K., and Yokoyama, K., A remark on the almost global existence theorems of Keel, Smith and Sogge, to appear in Funkcialaj Ekvacioj, 2005.

[7] Hörmander, L., Lectures on Nonlinear Hyperbolic Differential Equations, Math. Appl., 26, Springer, 1997.

[8] Keel, M., Smith, H. and Sogge, C., Almost global existence for some semilinear wave equations, J. Anal. Math., 87 (2002), 265-279.

[9] - Almost global existence for quasilinear wave equations in three space dimensions, J. Amer. Math. Soc., 17 (2004), 109-153.

[10] Klainerman, S. and Nicolò, F., The Evolution Problem in General Relativity, Prog. Math. Phys., 25, Birkhäuser, 2003.

[11] Lindblad, H. and Rodniansky, I., Global existence for the Einstein vacuum equations in wave coordinates, Preprint, 2004.

[12] Matsuyama, T., Asymptotics for the nonlinear dissipative wave equation, Trans. Amer. Math. Soc., 355, (2003), 865-899.

[13] Metcalfe, J. L., Global existence for semilinear wave equations exterior to nontrapping obstacles, Houston Math. J., 30 (2004), 259-281.

[14] Mochizuki, K., Scattering theory for wave equations, Kinokuniya, Tokyo, 1984.

[15] Morawetz, C. S., Time decay for the nonlinear Klein-Gordon equation, Proc. Roy. Soc., A306 (1968), 291-296. 